\title{
Satiety dysfunction in Prader-Willi syndrome demonstrated by FMRI
}

\author{
N A Shapira, M C Lessig, A G He, G A James, D J Driscoll, Y Liu
}

J Neurol Neurosurg Psychiatry 2005;76:260-262. doi: 10.1136/jnnp.2004.039024

The neurobiology relating to the insatiable appetite observed in Prader-Willi syndrome (PWS) has not been fully characterised. Two functional magnetic resonance imaging (fMRI) scans were performed on each of three adults with PWS. The scans were carried out pre- and post-treatment with the antiepileptic topiramate, which had little effect on body weight and appetite in these subjects. Subjects fasted overnight and drank a $75 \mathrm{~g}$ dextrose solution prior to fMRI scans for measurement of brain activation levels during/after glucose ingestion. Following glucose administration, there was a significant delay in activation at the hypothalamus and other brain regions associated with satiety compared with previous data on obese volunteers. These regions include the insula, ventromedial prefrontal cortex, and nucleus accumbens. Individuals with PWS showed a mean latency of $24 \mathrm{~min}$ while in a previous study obese volunteers had shown a latency of $15 \mathrm{~min}$ and lean volunteers a latency of $10 \mathrm{~min}$ in the hypothalamus. Our results provide evidence towards a satiety dysfunction in the central nervous system of PWS patients.

$\mathrm{P}$ rader-Willi syndrome (PWS) is a neurogenetic disorder characterised by hyperphagia that develops within the first 6 years of life following a period of failure to thrive. ${ }^{1}$ The insatiable appetite exhibited often leads to uncontrollable obesity in this population. ${ }^{1}$ Van Hooren et al have promoted control of obesity with preventative learning measures; however, token economies and the use of regulated exercise in group home settings appears to be the most efficacious treatment for the obesity associated with PWS. ${ }^{23}$ Some success was reported with the use of appetite suppressants such as fenfluramine, prior to their removal from the market, and in Japan the anorectic mazindol was shown to be beneficial in five individuals with PWS.$^{4-7}$ The aetiology of obesity has long been in question in these individuals, most often being attributed to satiety dysfunction. ${ }^{8}$ Del Parigi et al attribute elevated circulating ghrelin levels to the increased caloric intake in PWS individuals. ${ }^{9}$

To date, functional imaging of individuals with PWS has not been reported in the literature; however, several reports have shown abnormal cortical and adipose tissue via whole body magnetic resonance imaging. ${ }^{11}{ }^{11}$ Abnormal brain responses following food intake have been shown to be associated with obesity. ${ }^{12}{ }^{13}$ Recently, evidence has accumulated that differential brain responses to hunger and satiation have been identified across populations. ${ }^{14}{ }^{15}$ Both positron emission tomography and functional magnetic resonance imaging (fMRI) have shown investigational neural substrates of satiation in healthy and obese subjects. ${ }^{16}{ }^{17}$ Moreover, a new analysis model has been developed for measuring the dynamic correlation between neural and hormonal signals in clinical populations. ${ }^{18}$ In the current study, we applied this new method, temporal clustering analysis (TCA), to scan subjects with PWS based on a well controlled fMRI paradigm. ${ }^{8}$

\section{METHODS AND MATERIALS Subjects}

The study consisted of three adults with PWS (two females, 36 and 38 years of age and one male, 25 years of age; body mass index of $31.8,30.4$, and 37.8 respectively at enrolment). Participants had been confirmed as having PWS through chromosomal and DNA molecular analyses. Concomitant psychotropic medications at the time of enrolment included fluoxetine and valproic acid in the male subject and venlafaxine in the two female subjects. In addition to these medications, the subjects were taking vitamin and mineral supplements such as calcium. None of these individuals had been prescribed human growth hormone previously and they did not have a history of appetite suppressant use. These individuals provided full informed consent prior to study procedures in accordance with the institutional review board of the University of Florida Health Science Center. All three subjects were concurrently enrolled in an 8 week open label trial of the anticonvulsant topiramate for appetite regulation. ${ }^{19}$ No effect on food intake and appetite change was shown in this 8 week trial.

\section{MRI procedures}

For the reported fMRI study, the subjects were scanned in a 3.0 T MRI scanner (GE/Signa) with an fMRI protocol described previously. ${ }^{17}$ Briefly, functional images were obtained before ( 5 minutes for baseline) and after (for 37 minutes) a subject consumed a beverage containing $75 \mathrm{~g}$ dextrose, as in an oral glucose tolerance test. An echo planar imaging (EPI) sequence was used (echo time $25 \mathrm{~ms}$, repetition time $5000 \mathrm{~ms}$, flip angle $90^{\circ}$, matrix size $128 \times 128$; field of view $240 \times 240 \mathrm{~mm}^{2}$ ), with 24 axial slices (thickness $6 \mathrm{~mm}$ ) covering the whole brain. Tl weighted structural MR images were acquired before or after each functional scan with the same field of view and slice profile for the image co-registration with the EPI images and a conventional three-dimensional MRI. Subjects were given the glucose solution and scanned both prior to (scan 1 ) and post (scan 2 ) the 8 week open label trial with topiramate.

\section{Biochemical and appetite measurement}

Serial blood draws were carried out before, during, and after functional scans to measure glucose and insulin on all subjects. The measured blood glucose and insulin levels were within the normal range.

Abbreviations: $\mathrm{EPI}$, echo planar imaging; fMRI, functional magnetic resonance imaging; PWS, Prader-Willi syndrome; ROI, region of interest; TCA, temporal clustering analysis 
Image processing and data analysis

For each subject, the EPI images were analysed using TCA to identify the temporal maxima of the fMRI signal. ${ }^{17}$ The TCA method was used to extract the time course of global brain response, which is independent of the variance of individual anatomy. TCA measures the magnitude of the dynamic fMRI signal by forming a high density histogram of the image voxels that reach the maximum change at the time of imaging. After creating a time window in TCA, the EPI images acquired during this time window were compared with those acquired during the 5 min baseline using group $t$ tests. For each functional scan, the imaging data were then averaged over the three PWS subjects and a $t$ threshold applied based on a spatial clustering technique. ${ }^{20}$ Given the small sample size in the study, we chose a $t$ threshold reflecting statistical significance at $\mathrm{p}<0.01$ (uncorrected) for the voxel-wise changes in fMRI signal following glucose

A

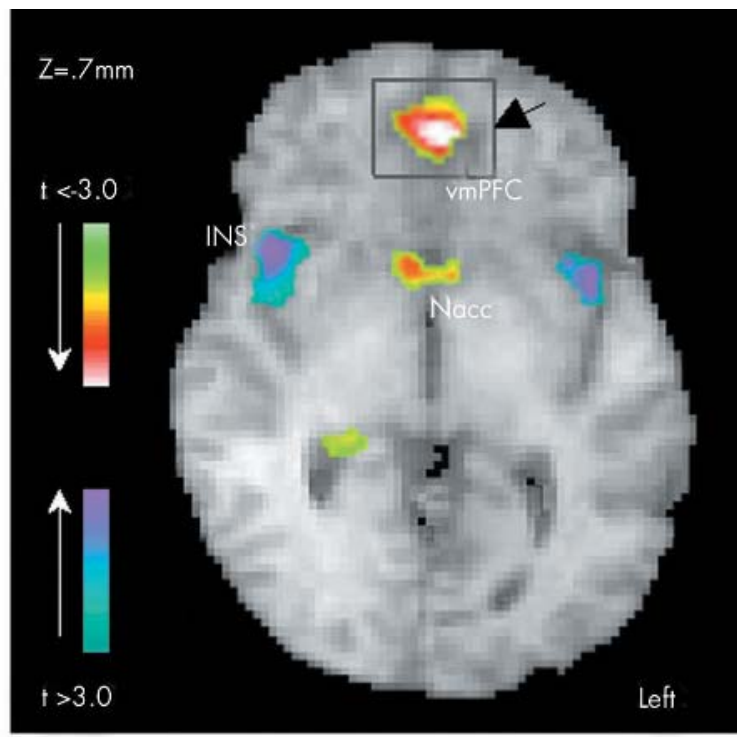

B

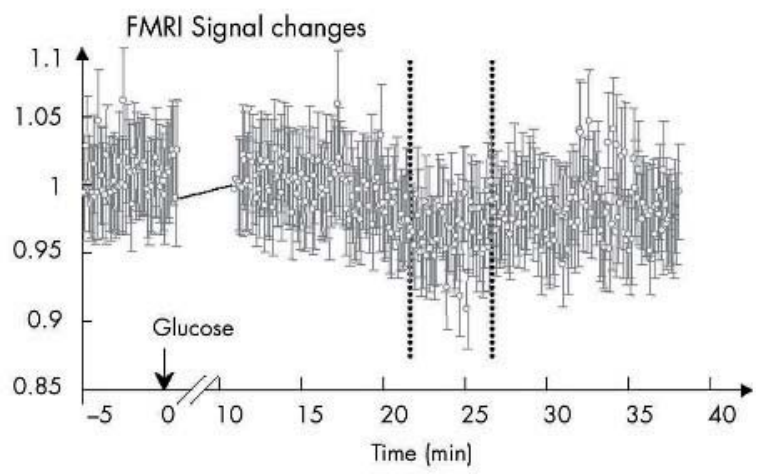

Figure 1 (A) A functional map showing brain activity changes in an axial brain slice $(z=-7 \mathrm{~mm})$ after oral glucose ingestion in an individual with Prader-Willi syndrome. The colour coded fMRI signal changes were determined by $t$ tests comparing the images acquired (22nd to 26th minute) after glucose administration with those acquired during the 5 minute baseline and overlaid on the corresponding structure MRI. A $t$ threshold of -3.0 and 3.0 was chosen to reflect a statistical significance level ( $p<0.01$, uncorrected) of the decrease and increase respectively of the fMRI signal after glucose administration. INS, insula; vmPFC, ventromedial prefrontal cortex; Nacc, nucleus accumbens. (B) Time course of fMRI signal (mean (SEM), $n=6$ ) at the vmPFC, showing a decrease of brain activity (relative to the baseline before glucose ingestion) that peaked around the 24th minute after glucose administration. The broken lines on the $\mathrm{X}$ axis indicate a period for the subject to finish the drink and the time of restarting the MR scan. administration. The resulting statistic parametric maps were co-registered with the $\mathrm{Tl}$ weighted images for Talairach normalisation $^{21}$ and localisation. The regions of interest (ROIs) were identified as those above threshold areas of functional activations. These ROIs were used for further analysis of the time courses of the local fMRI signal. ${ }^{22}$

\section{RESULTS}

Dynamic analysis using TCA demonstrated that maximum changes in brain activity appeared approximately 24 minutes (23.8 (4.3) min for scan 1; 24.04 (3.7) min for scan 2) after oral glucose administration. Fig 1 shows that a 5 min time window centred at the 24th min was generated for mapping the fMRI signal change compared with the baseline. For both times (i.e., pre- and post-topiramate treatment), the ROI analysis showed negative changes in the ventromedial prefrontal cortex, the nucleus accumbens, and the hypothalamus, and positive changes in the dorsolateral prefrontal cortex and insula (fig 1A). Given the small number of subjects, it should be noted that the ventromedial prefrontal cortex is the only region remaining on the activation map if a corrected statistical threshold is used for multiple comparisons. The time course of fMRI signal changes was extracted from this region and averaged across the six scans performed on the three subjects (fig $1 \mathrm{~B}$ ).

There was no significant difference in the delays and magnitudes of brain activity changes before and after topiramate treatment based on direct comparisons between scans 1 and 2 (images not shown), except for a trend towards increase of insular activation after treatment. Topiramate therapy did not significantly alter body mass index or appetite as measured by a "feelings of hunger" visual scale, ${ }^{19}$ which was consistent with the imaging data.

\section{DISCUSSION}

Our preliminary data demonstrate a delay of 24 min for PWS brain activity after glucose administration. In a previous fMRI study using a single sagittal slice, healthy lean subjects showed changes around 10 minutes and obese subjects around 15 minutes in the hypothalamus. ${ }^{12}{ }^{17}$ With imaging of the whole brain, the current study has identified the brain regions, including the insular cortex, the prefrontal cortex, the ventral basal ganglia, and the hypothalamus, which have a consistent delay in response to glucose ingestion. These brain regions consist of a distributed functional network, which has been implicated in the regulation of hunger and satiation. ${ }^{16}$ We also observed that the delay time in brain response in PWS to glucose ingestion was not altered by topiramate.

Limitations of this study are the small number of subjects, and, as previous fMRI studies ${ }^{12}{ }^{17}$ acquired only a single sagittal slice, PWS and obese subjects might show a similar brain activation pattern in areas outside of the hypothalamus.

In conclusion, our results provide further evidence towards a satiety dysfunction in the central nervous system of PWS patients. Future studies will be needed to fully characterise this dysfunction and the endocrine contribution to it.

\section{ACKNOWLEDGEMENTS}

This work was supported by the Prader-Willi Syndrome Association (USA) and National Institutes of Health (NS45518 to Y Liu).

\section{Authors' affiliations}

N A Shapira, A G He, G A James, Y Liu, Department of Psychiatry, University of Florida, Gainesville, FL, USA

D J Driscoll, Department of Pediatrics, University of Florida, Gainesville, FL, USA 
M C Lessig, Department of Psychiatry \& Behavioral Sciences, University of Washington, Seattle, WA, USA

Competing interests: none declared

The work was presented in part at the $24^{\text {th }}$ Annual National Conference of the Prader-Willi Syndrome Association (USA) Scientific Conference, Salt Lake City, July 11, 2002.

Correspondence to: Dr N A Shapira, P.O. Box 100256, Gainesville, FL 32610-0256, USA; shapira@psychiatry.ufl.edu

Received 9 February 2004

In revised form 6 April 2004

Accepted 7 April 2004

\section{REFERENCES}

1 Holm VA, Cassidy SB, Butler MG, et al. Prader-Willi syndrome: Consensus diagnostic criteria. Pediatrics 1993;91:398-402.

2 Van Hooren RH, Widdershoven GAM, van der Borne HW, et al. Autonomy and intellectual disability: the case of prevention of obesity in Prader-Willi syndrome. J Intell Dis Res 2002;46:560-8.

3 Page TJ, Stanley AE, Richman GS, et al. Reduction of food theft and long-term maintenance of weight loss in a Prader-Willi adult. J Behav Ther Exp Psychiatry 1983;14:261-8.

4 Selikowitz M, Sunman J, Pendergast A, et al. Fenfluramine in Prader-Willi syndrome: a double blind, placebo controlled trial. Arch Dis Child 1990;65:112-14

5 Inove S. Clinical studies with mazindol. Obes Res 1995;(Suppl 4):549-52S

6 Itoh K, Koeda T, Ohno K, et al. Effects of mazindol in two patients with PraderWilli syndrome. Pediatr Neurol 1995; 13:349-51.

7 Invi A, Uemoto M, Takamiya S, et al. A case of Prader-Willi syndrome with long-term mazindol treatment (Letter). Arch Intern Med 157:464.
8 Lindgren AC, Barkeling B, Hagg A, et al. Eating behavior in Prader-Willi syndrome, normal weight, and obese groups. J Pediatr 2000;137:50-5.

9 Del Parigi A, Tschop M, Heiman ML, et al. High circulating ghrelin: a potential cause for hyperphagia and obesity in Prader-Willi syndrome. J Clin Endocrinol Metab 2002;87:5461-4.

10 Yoshii A, Krishnamoorthy KS, Grant PE. Abnormal cortical development shown by 3D MRI in Prader-Willi syndrome. Neurology 2002;59:644-5.

11 Goldstone AP, Thomas EL, Brynes AE, et al. Visceral adipose tissue and metabolic complications of obesity are reduced in Prader-Willi syndrome female adults: evidence for novel influences on body fat distribution. J Clin Endocrinol Metab 2001;86:4330-8.

12 Matsuda M, Liu Y, Mahankali S, et al. Altered hypothalamic response to oral glucose intake in obese humans. Diabetes 1999:48:1801-6.

13 Gautier JF, Chen K, Salbe AD, et al. Differential brain responses to satiation in obese and lean men. Diabetes 2000;49:838-46.

14 Gautier JF, Del Parigi A, Chen K, et al. Effect of satiation on brain activity in obese and lean women. Obes Res $2001 ; 9: 676-84$

15 Del Parigi A, Chen K, Gautier JF, et al. Sex differences in the human brain's response to hunger and satiation. Am J Clin Nutr 2002;75:1017-22.

16 Tataranni PA, Gautier JF, Chen K, et al. Neuroanatomical correlates of hunger and satiation in humans using positron emission tomography. Proc Natl Acad Sci USA 1999;96:4569-74.

17 Liv Y, Gao JH, Liu HL, et al. The temporal response of the brain after eating revealed by functional MRI. Nature 2000;405:1058-62.

18 James GA, Guo W, Liu Y. Imaging in vivo brain-hormone interaction in the control of eating and obesity. Diabetes Technol Ther 2001;3:617-22.

19 Shapira NA, Lessig MC, Lewis MH, et al. Effects of topiramate in adults with Prader-Willi syndrome. Am J Ment Retard 2004;109:299-307.

20 Xiong J, Gao J-H, Lancaster JL, et al. Clustered pixels for functional MRI activation studies in human brain. Hum Brain Mapp 1995:3:210-25.

21 Talaraich J, Tournoux P. Co-planar stereotaxic atlas of the human brain. New York: Thieme Medical Publisher, 1988.

22 Liu Y, Gao JH, Liotti M, et al. Fox PT: Temporal dissociation of parallel processing in the human subcortical outputs. Nature 1999;400:364-67. 